$\Phi=$ 盎

\title{
Atherosclerotic disease and diabetes mellitus
}

\author{
Malyn Martha Lilac Ketisha Antoine ${ }^{1}$, Yancheng Xu ${ }^{1}{ }^{*}$, Dodji Kossi Djakpo ${ }^{1}$ \\ ${ }^{1}$ Department of Endocrinology, Zhongnan Hospital of Wuhan University, Hubei, China \\ *Corresponding author E-mail: xjl100901@whu.edu.cn
}

\begin{abstract}
The macro-vascular complications (cardiovascular, neurovascular and peripheral vascular diseases) observed in diabetic patients usually develop secondary to the presence of atherosclerotic diseases. In diabetic patients chronically elevated blood glucose levels play a major role in contributing to the development of the disease; however, in addition to hyperglycaemia, other factors such as hypertension, dyslipidaemia and obesity also contribute to the development of this condition. Despite the fact that further research is required to fully establish the relationship between hyperglycaemia and the development of atherosclerotic plaques in diabetic patients, three major pathways have already been identified for the role they play in the pathogenesis of atherosclerosis. These pathways are identified as follows: 1. nonenzymatic glycosylation of lipids and proteins 2. oxidative stress 3.Protein kinase C. This review discusses how hyperglycaemia influences the development of atherosclerosis which further leads to the development of major vascular complications in diabetic patients.
\end{abstract}

Keywords: Atherosclerosis; Diabetes Mellitus (DM); Hyperglycaemia.

\section{Introduction}

In places like the United States of America (USA), the number of observed cases of diabetes mellitus (DM) continues to rise at alarming rates (Blonde, 2010). Diabetes significantly increases the risk of developing coronary heart diseases (CHD) and diabetic patients who develop CHD are three to seven times more likely to die from coronary complications (Haffner et al. 1998). Emerging evidence from research has demonstrated that the presence of diabetes is associated with an increased risk for developing atherosclerosis and this evidence further highlights that atherosclerosis in diabetic patients is one of the major risk factors associated with the development of cardiovascular diseases (CVD) (Zheng et al. 2019) and other macro-vascular complications (Ross, 1988). Studies further demonstrate that the process involved in the development of atherosclerosis and its clinical presentations depend significantly on the vessels affected (Poredos et al. 2018).

In addition to exhibiting abnormally high blood glucose levels, diabetic patients may also show signs of hypertension, high cholesterol, and obesity. These additional factors cumulatively accelerate the development of atherosclerosis which in turn leads to the development of the vascular complications that are often associated with diabetes (Saito et al. 2000). In diabetic patients, the presence of chronically elevated blood glucose levels is found to be a strong contributor to the development of vascular complications secondary to atherosclerotic diseases. Research such as "The Nurses' Health Study" conducted in the United States demonstrates that as compared to the general population, diabetic patients experience higher rates of CVD (Nesto, 2004).

In light of the increased risks of vascular complications observed in diabetic patients, this review serves to examine the association between $\mathrm{DM}$ and the development of atherosclerotic plaques further leading to the development of cardiovascular diseases and other macro-vascular complications commonly observed in diabetic patients.

\section{Atherosclerosis in diabetes}

Diabetes is a metabolic disorder characterized mainly by abnormally high blood glucose levels accompanied by abnormal blood lipid profiles and insulin resistance. Collectively these factors impair the normal functions of blood vessels thus increasing the risk for developing vascular diseases (Association 2010, Alberti \& Zimmet 1998).

The endothelium lines blood vessels creating a barrier that permits the selective movements of substances between the blood and the tissues of the body. In addition to serving homeostatic and immunological functions, the endothelium also regulates blood flow and oxygen delivery to underlying tissues (Davies \& Hagen 1993). Physiologically the endothelium produces various substances such as prostaglandins, endothelin and nitric oxide (NO). NO plays an important role as it functions to suppress platelet activation, reduce the migration and proliferation of vascular smooth muscle cells and prevent inflammation (Williams et al. 1996, Beckman et al. 2002).

The presence of hyperglycaemia as seen in diabetic patients, fuels a series of mechanisms that impairs the normal functioning of endothelial cells which either reduces the production of important vasodilators such as NO or promotes the production of vasoconstrictors such as endothelin-1(Stehouwer et al. 1997). In addition, hyperglycaemia significantly affects the normal functioning of platelets and vascular smooth muscles cells ${ }^{12}$. Therefore, the overall effects of prolonged elevated blood glucose levels promote the development of atherosclerotic plaques which in turn contribute to varying forms of vascular diseases observed in diabetic patients. 


\subsection{Hyperglycaemia and atherosclerosis}

It is currently established that prolonged elevated blood glucose levels (hyperglycaemia) play a major role in the atherosclerotic process as is observed in diabetic patients (Control \& Group 1993). Both animal and human studies have demonstrated the involvement of three major pathways in the pathogenesis of atherosclerosis in diabetes. These pathways include the following: 1) non-enzymatic glycosylation of lipids and proteins 2) oxidative stress and 3) protein kinase C (PKC) activation (Nishikawa et al. 2000, Aronson \& Rayfield 2002). Evidence obtained in bovine endothelial cells culture suggests that hyperglycaemia rapidly increases reactive oxygen species production (Giardino et al. 1996). It has also been reported in vitro that high glucose levels have the potential to promote apoptosis thus causing endothelial dysfunction leading to the development of atherosclerosis (Baumgartner-Parzer et al. 1995). In vitro studies demonstrated the close relationship between high blood glucose and atherosclerosis; however, these studies still lack crucial evidence.

Animal experimental studies have elucidated a relationship between glucose and lipids in the process of atherosclerosis. Thus, one large study conducted in pigs demonstrated that the presence of diabetes accelerates atherosclerosis in the presence of Hyperlipidaemia (Gerrity et al. 2001). Hyperglycaemia alone gives the impression of being insufficient to accelerate the atherosclerotic process even though it might play an important role in this phenomenon. The glucose is likely to act with lipid to accelerate atherosclerosis in diabetes (Kanter et al. 2007).

\section{Complications of atherosclerosis in diabetes}

Chronic complications of DM can be categorized as either micro-vascular or macro-vascular in nature. Micro-vascular complications follow chronically elevated blood glucose levels and include nephropathy, retinopathy and peripheral neuropathy (Nathan 1993, Reusch 2003). On the contrary, macro-vascular complications are preceded by the development of atherosclerotic plaques which then lead to cardiovascular, neurovascular or peripheral vascular diseases (Fowler, 2008).

Diabetes is an independent risk factor for several CVD. In both genders, CVD is the main cause of death in approximately $65 \%$ of people living with DM (Wilson Peter W. F. et al. 1998, Deshpande et al. 2008). In general, patients with atherosclerotic CHD and diabetes experience higher rates of mortality. It is reported that DM is likely an independent risk factor for atherosclerotic coronary artery disease (McGill \& McMahan 1998, Wilson, 1998). Moreover, in patients with diabetes, multi-vessel atherosclerosis is often associated with myocardial ischemia and can be sometimes asymptomatic (Wingard et al. 1993). Atherosclerotic CHD is associated with the worst prognosis in diabetic patients.

Data suggest that hypertension, cigarette smoking, and high serum cholesterol constitute covariate cardiovascular risk factors in diabetic patients (Stone et al. 1989, Stamler et al. 1993, 'Intensive blood-glucose control with sulphonylureas or insulin compared with conventional treatment and risk of complications in patients with type 2 diabetes (UKPDS 33)' 1998). Moreover, physical inactivity, sex, obesity, advancing age, and genetics are considered to be predisposing risk factors that affect the development of diabetes and CVD. It is well known that hypertension is one of the major risk factors for stroke and coronary artery disease. However, in diabetes, arterial hypertension plays an important role by leading to diabetic nephropathy (Grundy Scott M. et al. 1999). Thus, the risk of CVD increases in patients with high blood pressure and diabetes. Diabetic renal impairment in both types $1 \& 2$ diabetes mellitus is a severe complication that increases the risk of mortality. Overall, the leading cause of death in these patients is CVD (Rischen-Vos et al. 1992, Aubert 1995). In diabetic patients with CHD, myocardial dysfunction often contributes to diabetic cardiomyopathy (Spector, 1998). It is reported that many factors such as chronic high blood sugar, prolonged hypertension and severe coronary atherosclerosis also may lead to the development of diabetic cardiomyopathy (Mahgoub \& Abd-Elfattah 1998).

Diabetes is often associated with cerebrovascular diseases such as strokes. Diabetic patients are most likely at a higher risk of developing a stroke than non-diabetic patients. Evidence shows that diabetic patients aged 20 to 65 demonstrate a 5 times higher risk for developing a stroke as compared to non-diabetic patients (Papatheodorou et al. 2016). In addition, diabetes increases cerebrovascular mortality rates (Diabetes in America, 3rd Edition | NIDDK, n.d.). Carotid atherosclerosis is often found in diabetic patients and the carotid intima-media thickness is used as a predictor of vascular risk in diabetic patients with coronary artery disease (Djaberi et al. 2009).

Diabetic patients are also at risk of developing peripheral vascular diseases. Many factors have been proposed for the development of diabetes-associated peripheral arterial diseases which include, age and gender. As age increases the risk for developing this condition also increases in both type 1 and type 2 diabetic patients (Elnady \& Saeed 2017). The Framingham and Rochester studies demonstrated that men were more likely to develop peripheral vascular diseases than women; however, these studies also revealed that diabetic women are more likely to develop peripheral vascular complications as compared to non-diabetic women (Jude et al. 2010). Peripheral vascular diseases can also lead to the development of atherosclerotic foot ulcers causing intermittent claudication and commonly resulting in leg amputations. Due to the fact that it is often present along with CVD, ischemic foot ulcers usually carry an overall poor prognosis (Henry, 1987).

\section{Conclusion}

Although a strong correlation has been established between prolonged elevated blood glucose levels and the development of atherosclerosis, other major factors such as hypertension, dyslipidaemia and cigarette smoking have also been proven to play major roles in the development and progression of the condition. Further studies are required to fully explain the role that hyperglycaemia plays in the process of atherosclerosis in diabetes as in vitro studies have proven in this regard to be inconclusive.

\section{References}

[1] Alberti, K. G. M. M., \& Zimmet, P. Z. (1998). Definition, diagnosis and classification of diabetes mellitus and its complications. Part 1: Diagnosis and classification of diabetes mellitus. Provisional report of a WHO consultation. Diabetic Medicine, 15(7), 539-553. https://doi.org/10.1002/(SICI)1096-9136(199807)15:7<539::AID-DIA668>3.0.CO;2-S.

[2] Aronson, D., \& Rayfield, E. J. (2002). How hyperglycemia promotes atherosclerosis: Molecular mechanisms. Cardiovascular Diabetology, 1(1), 1. https://doi.org/10.1186/1475-2840-1-1.

[3] Association, A. D. (2010). Diagnosis and Classification of Diabetes Mellitus. Diabetes Care, 33(Suppl 1), S62. https://doi.org/10.2337/dc10-S062.

[4] Aubert, R. (1995). Diabetes in America. DIANE Publishing. 
[5] Baumgartner-Parzer, S. M., Wagner, L., Pettermann, M., Grillari, J., Gessl, A., \& Waldhäusl, W. (1995). High-glucose-triggered apoptosis in cultured endothelial cells. Diabetes, 44(11), 1323-1327. https://doi.org/10.2337/diab.44.11.1323.

[6] Beckman, J. A., Creager, M. A., \& Libby, P. (2002). Diabetes and atherosclerosis: Epidemiology, pathophysiology, and management. Jama, 287(19), 2570-2581. https://doi.org/10.1001/jama.287.19.2570.

[7] Blonde, L. (2010). Current antihyperglycemic treatment guidelines and algorithms for patients with type 2 diabetes mellitus. The American Journal of Medicine, 123(3), S12-S18. https://doi.org/10.1016/j.amjmed.2009.12.005.

[8] Control, D., \& Group, C. T. R. (1993). The effect of intensive treatment of diabetes on the development and progression of long-term complications in insulin-dependent diabetes mellitus. New England Journal of Medicine, 329(14), 977-986. https://doi.org/10.1056/NEJM199309303291401.

[9] Davies, M. G., \& Hagen, P.-O. (1993). The vascular endothelium. A new horizon. Annals of Surgery, 218(5), 593. https://doi.org/10.1097/00000658199321850-00003

[10] Deshpande, A. D., Harris-Hayes, M., \& Schootman, M. (2008). Epidemiology of Diabetes and Diabetes-Related Complications. Physical Therapy, 88(11), 1254. https://doi.org/10.2522/ptj.20080020.

[11] Diabetes in America, 3rd Edition | NIDDK. (n.d.). National Institute of Diabetes and Digestive and Kidney Diseases. Retrieved 8 September 2019, from https://www.niddk.nih.gov/about-niddk/strategic-plans-reports/diabetes-in-america-3rd-edition.

[12] Djaberi, R., Schuijf, J. D., de Koning, E. J., Rabelink, T. J., Smit, J. W., Kroft, L. J. M., Pereira, A. M., Scholte, A. J., Spaans, M., Romijn, J. A., de Roos, A., van der Wall, E. E., Jukema, J. W., \& Bax, J. J. (2009). Usefulness of carotid intima-media thickness in patients with diabetes mellitus as a predictor of coronary artery disease. The American Journal of Cardiology, 104(8), 1041-1046. https://doi.org/10.1016/j.amjcard.2009.06.004.

[13] Elnady, B. M., \& Saeed, A. (2017). Peripheral Vascular Disease: The Beneficial Effect of Exercise in Peripheral Vascular Diseases Based on Clinical Trials. Advances in Experimental Medicine and Biology, 1000, 173-183. https://doi.org/10.1007/978-981-10-4304-8 11.

[14] Fowler, M. J. (2008). Microvascular and Macrovascular Complications of Diabetes. Clinical Diabetes, 26(2), 77-82. https://doi.org/10.2337/diaclin.26.2.77.

[15] Gerrity, R. G., Natarajan, R., Nadler, J. L., \& Kimsey, T. (2001). Diabetes-induced accelerated atherosclerosis in swine. Diabetes, 50(7), 1654-1665. https://doi.org/10.2337/diabetes.50.7.1654.

[16] Giardino, I., Edelstein, D., \& Brownlee, M. (1996). BCL-2 expression or antioxidants prevent hyperglycemia-induced formation of intracellular advanced glycation endproducts in bovine endothelial cells. The Journal of Clinical Investigation, 97(6), 1422-1428. https://doi.org/10.1172/JCI118563.

[17] Grundy Scott M., Benjamin Ivor J., Burke Gregory L., Chait Alan, Eckel Robert H., Howard Barbara V., Mitch William, Smith Sidney C., \& Sowers James R. (1999). Diabetes and Cardiovascular Disease. Circulation, 100(10), 1134-1146. https://doi.org/10.1161/01.CIR.100.10.1134.

[18] Haffner, S. M., Lehto, S., Rönnemaa, T., Pyörälä, K., \& Laakso, M. (1998). Mortality from coronary heart disease in subjects with type 2 diabetes and in nondiabetic subjects with and without prior myocardial infarction. New England Journal of Medicine, 339(4), 229-234. https://doi.org/10.1056/NEJM199807233390404.

[19] Henry, W. L. (1987). The Complications of Diabetes Mellitus. Journal of the National Medical Association, 79(6), 677-680.

[20] Intensive blood-glucose control with sulphonylureas or insulin compared with conventional treatment and risk of complications in patients with type 2 diabetes (UKPDS 33). (1998). The Lancet, 352(9131), 837-853. https://doi.org/10.1016/S0140-6736(98)07019-6.

[21] Jude, E. B., Eleftheriadou, I., \& Tentolouris, N. (2010). Peripheral arterial disease in diabetes-A review. Diabetic Medicine, 27(1), 4-14. https://doi.org/10.1111/j.1464-5491.2009.02866.x.

[22] Kanter, J. E., Johansson, F., LeBoeuf, R. C., \& Bornfeldt, K. E. (2007). Do glucose and lipids exert independent effects on atherosclerotic lesion initiation or progression to advanced plaques? Circulation Research, 100(6), 769-781. https://doi.org/10.1161/01.RES.0000259589.34348.74.

[23] Mahgoub, M. A., \& Abd-Elfattah, A. S. (1998). Diabetes mellitus and cardiac function. Molecular and Cellular Biochemistry, $180(1-2)$, 59-64. https://doi.org/10.1023/A:1006834922035.

[24] McGill, H. C., \& McMahan, C. A. (1998). Determinants of atherosclerosis in the young. American Journal of Cardiology, 82(10), 30-36. https://doi.org/10.1016/S0002-9149(98)00720-6.

[25] Nathan, D. M. (1993). Long-term complications of diabetes mellitus. New England Journal of Medicine, $328(23)$, 1676-1685. https://doi.org/10.1056/NEJM199306103282306.

[26] Nesto, R. W. (2004). Correlation between cardiovascular disease and diabetes mellitus: Current concepts. The American Journal of Medicine, 116(5), 11-22. https://doi.org/10.1016/j.amjmed.2003.10.016.

[27] Nishikawa, T., Edelstein, D., Du, X. L., Yamagishi, S., Matsumura, T., Kaneda, Y., Yorek, M. A., Beebe, D., Oates, P. J., \& Hammes, H.-P. (2000). Normalizing mitochondrial superoxide production blocks three pathways of hyperglycaemic damage. Nature, $404(6779)$, 787. https://doi.org/10.1038/35008121.

[28] Papatheodorou, K., Papanas, N., Banach, M., Papazoglou, D., \& Edmonds, M. (2016). Complications of Diabetes 2016. Journal of Diabetes Research, 2016, 6989453. https://doi.org/10.1155/2016/6989453.

[29] Poredos, P., Poredos, P., \& Jezovnik, M. K. (2018). Structure of Atherosclerotic Plaques in Different Vascular Territories: Clinical Relevance. Current Vascular Pharmacology, 16(2), 125-129. https://doi.org/10.2174/1570161115666170227103125.

[30] Reusch, J. E. (2003). Diabetes, microvascular complications, and cardiovascular complications: What is it about glucose? The Journal of Clinical Investigation, 112(7), 986-988. https://doi.org/10.1172/JCI200319902.

[31] Rischen-Vos, J., van der Woude, F. J., Tegzess, A. M., Zwinderman, A. H., Gooszen, H. C., van den Akker, P. J., \& van Es, L. A. (1992). Increased morbidity and mortality in patients with diabetes mellitus after kidney transplantation as compared with non-diabetic patients. Nephrology Dialysis Transplantation, 7(5), 433-437. https://doi.org/10.1093/oxfordjournals.ndt.a092162.

[32] Ross, R. (1988). Endothelial injury and atherosclerosis. In Endothelial cell biology in health and disease (pp. 371-384). Springer. https://doi.org/10.1007/978-1-4613-0937-6_17.

[33] Saito, I., Folsom, A. R., Brancati, F. L., Duncan, B. B., Chambless, L. E., \& McGovern, P. G. (2000). Nontraditional risk factors for coronary heart disease incidence among persons with diabetes: The Atherosclerosis Risk in Communities (ARIC) Study. Annals of Internal Medicine, 133(2), 8191. https://doi.org/10.7326/0003-4819-133-2-200007180-00007.

[34] Spector, K. S. (1998). Diabetic cardiomyopathy. Clinical Cardiology, 21(12), 885-887. https://doi.org/10.1002/clc.4960211205.

[35] Stamler, J., Vaccaro, O., Neaton, J. D., Wentworth, D., \& The Multiple Risk Factor Intervention Trial Research Group. (1993). Diabetes, Other Risk Factors, and 12-Yr Cardiovascular Mortality for Men Screened in the Multiple Risk Factor Intervention Trial. Diabetes Care, 16(2), 434-444. https://doi.org/10.2337/diacare.16.2.434.

[36] Stehouwer, C. D., Lambert, J., Donker, A. J. M., \& van Hinsbergh, V. W. (1997). Endothelial dysfunction and pathogenesis of diabetic angiopathy. Cardiovascular Research, 34(1), 55-68. https://doi.org/10.1016/S0008-6363(96)00272-6.

[37] Stone, P. H., Muller, J. E., Hartwell, T., York, B. J., Rutherford, J. D., Parker, C. B., Turi, Z. G., Strauss, H. W., Willerson, J. T., Robertson, T., Braunwald, E., \& Jaffe, A. S. (1989). The effect of diabetes mellitus on prognosis and serial left ventricular function after acute myocardial infarction: Contribution of both coronary disease and diastolic left ventricular dysfunction to the adverse prognosis. Journal of the American College of Cardiology, 14(1), 49-57. https://doi.org/10.1016/0735-1097(89)90053-3.

[38] Williams, S. B., Cusco, J. A., Roddy, M.-A., Johnstone, M. T., \& Creager, M. A. (1996). Impaired nitric oxide-mediated vasodilation in patients with non-insulin-dependent diabetes mellitus. Journal of the American College of Cardiology, 27(3), 567-574. https://doi.org/10.1016/07351097(95)00522-6

[39] Wilson, P. W. (1998). Diabetes mellitus and coronary heart disease. American Journal of Kidney Diseases, 32(5), S89-S100. https://doi.org/10.1053/ajkd.1998.v32.pm9820468.

[40] Wilson Peter W. F., D’Agostino Ralph B., Levy Daniel, Belanger Albert M., Silbershatz Halit, \& Kannel William B. (1998). Prediction of Coronary Heart Disease Using Risk Factor Categories. Circulation, 97(18), 1837-1847. https://doi.org/10.1161/01.CIR.97.18.1837. 
[41] Wingard, D. L., Barrett-Connor, E. L., Scheidt-Nave, C., \& McPhillips, J. B. (1993). Prevalence of Cardiovascular and Renal Complications in Older Adults With Normal or Impaired Glucose Tolerance or NIDDM: A population-based study. Diabetes Care, 16(7), 1022-1025. https://doi.org/10.2337/diacare.16.7.1022.

[42] Zheng, L.-Y., Xu, X., Wan, R.-H., Xia, S., Lu, J., \& Huang, Q. (2019). Association between serum visfatin levels and atherosclerotic plaque in patients with type 2 diabetes. Diabetology \& Metabolic Syndrome, 11(1), 60. https://doi.org/10.1186/s13098-019-0455-5. 